\title{
A prospective phase-Il trial of biweekly docetaxel plus androgen deprivation therapy in patients with previously-untreated metastatic castration-naïve prostate cancer
}

Seonggyu Byeon ${ }^{1 \dagger}$, Hongsik Kim² ${ }^{2 \dagger}$, Hwang Gyun Jeon ${ }^{3}$, Seong II Seo ${ }^{3}$, Seong Soo Jeon ${ }^{3}$, Hyun Moo Lee ${ }^{3}$, Soon II Lee ${ }^{4}$ and Se Hoon Park ${ }^{2^{*}}$

\begin{abstract}
Introduction: The aim of this prospective phase II study was to evaluate the efficacy and safety of biweekly docetaxel plus androgen-deprivation therapy (ADT) in patients with metastatic castration-naïve prostate cancer (mCNPC).

Patients and methods: Patients with histologically-proven, previously-untreated mCNPC received ADT plus docetaxel, $40 \mathrm{mg} / \mathrm{m}^{2}$. Docetaxel was repeated every 2 weeks, up to 12 cycles. Endpoints included castration-resistant prostate cancer (CRPC)-free survival, prostate-specific antigen (PSA) response, and safety.

Results: A total of 42 patients were registered and analyzed for final outcomes. Of the 42 patients, 36 (86\%) completed the 12 planned cycles of docetaxel plus ADT. During a median follow up of 25 months, all but two patients (95\%) achieved a PSA response with a nadir PSA level of $0.42 \mathrm{ng} / \mathrm{ml}$ (range $0.01-1280.87$ ). The median CRPC-free survival was 26.4 months (95\% confidence interval [CI] 20.9-32.0) with a one-year CRPC-free rate of $79 \%$ (33 patients, 95\% Cl 66-91). Multivariable analysis revealed that the performance status of the Eastern Cooperative Oncology Group 0 was independently associated with longer CRPC-free survival (hazard ratio [HR] 0.27, 95\% Cl 0.07-0.99). The most common adverse events of any grade were anemia (95\%), followed by nail changes (33\%), fatigue (29\%), and oral mucositis (26\%). Severe (grade 3 or higher) adverse events were infrequent: pneumonitis $(n=2)$, diarrhea $(n=1)$, and neutropenia $(n=1)$.
\end{abstract}

Conclusion: Our results suggest that biweekly docetaxel plus ADT is feasible, and clinical efficacy does not seem to be compromised compared to a standard triweekly docetaxel $75 \mathrm{mg} / \mathrm{m}^{2}$ plus ADT regimen.

\section{Introduction}

Globally,prostate cancer is a leading cause of cancerrelated deaths, with more than 10,000 cases diagnosed and 1821 deaths annually in Korea alone [1]. Prostate

\footnotetext{
*Correspondence: hematoma@skku.edu

${ }^{\dagger}$ Seonggyu Byeon and Hongsik Kim contributed equally to this work.

${ }^{2}$ Division of Hematology-Oncology, Department of Medicine,

Sungkyunkwan University School of Medicine, Samsung Medical Center,

Seoul, South Korea

Full list of author information is available at the end of the article
}

cancer is an androgen-dependent disease, and the main treatment in the control of prostate cancer growth is androgen-deprivation therapy (ADT), including a luteinizing hormone-releasing hormone (LHRH) agonist/ antagonist (medical castration) or bilateral orchiectomy (surgical castration) [2]. Based on findings from recent clinical trials [3-5], current guidelines have established the addition of docetaxel or modern androgen axis targeting agents (abiraterone acetate, and enzalutamide) to $\mathrm{ADT}$ as the standard of care for patients with metastatic 
castration-naïve prostate cancer (mCNPC) $[6,7]$. While the evidence is compelling when analyzed by the volume of disease or risk, the long term follow-up of the largest trial confirmed the benefit of adding docetaxel to ADT persisted regardless of disease burden [8].

A major challenge in the management of $\mathrm{mCNPC}$ is balancing the toxicity of therapy with clinical benefit. In most trials involving docetaxel, patients received 6 cycles at $75 \mathrm{mg} / \mathrm{m}^{2}$ every 3 weeks with ADT. Although the triweekly regimen was active to $\mathrm{mCNPC}[3,4]$, relatively high incidence of febrile neutropenia was regarded as one of the major obstacles. As an alternative treatment, a weekly or biweekly docetaxel administration has been considered as a way of attractive regimen with reduced hematological toxicity.

In a phase III trial comparing docetaxel $50 \mathrm{mg} / \mathrm{m}^{2}$ every other week to docetaxel $75 \mathrm{mg} / \mathrm{m}^{2}$ every third week in metastatic castration-resistant prostate cancer (mCRPC) [9], the biweekly regimen was associated with reduced hematological toxicities, fatigue being the most frequent adverse event, and a significantly longer time to treatment failure and improved overall survival, which may be related to a greater dose-intensity. These findings, supported by our retrospective studies performed in mCRPC as well as in $\mathrm{mCNPC}$ settings, suggest that the tolerability of docetaxel could be further improved [10,11]. We thus designed the present prospective phase 2 study to evaluate the safety and efficacy of a biweekly schedule of docetaxel added to ADT as first-line treatment in $\mathrm{mCNPC}$.

\section{Patients and methods}

\section{Patients}

In the present single-center, prospective phase II study, key eligibility criteria included histologically confirmed mCNPC. Other inclusion criteria were as follows: age 20 years or older; an Eastern Cooperative Oncology Group (ECOG) performance status of 0 or 1 ; no prior systemic treatment; at least one measurable metastatic lesion or evaluable bone lesions; and adequate organ function. Eligible patients were selected by a multidisciplinary urologic oncology tumor board composed of urologists, radiologists, pathologists, and medical oncologists. The study protocol (ClinicalTr ials.gov, NCT03061643 [23/02/2017]; CRIS.nih.go.kr, KCT0003546) was approved by the institutional review board of Samsung Medical Center (SMC 2017-01005-005, Seoul, Korea). All patients provided written informed consent. All study procedures were conducted in accordance to good clinical practice, and the Declaration of Helsinki.

\section{Treatment and evaluation}

Following 4weeks of ADT treatment, patients received docetaxel $40 \mathrm{mg} / \mathrm{m}^{2}$ intravenously on day 1 with standard premedication. Docetaxel was repeated every 2 weeks, for up to 12 cycles in the absence of unacceptable toxicity or progressive disease. Supportive care, including the administration of anti-emetics, blood products, bisphosphonates or denosumab, and the use of analgesics, was given if judged appropriate by the investigator. Primary granulocyte colony stimulating factor (G-CSF) prophylaxis was not permitted unless the patient had history of febrile neutropenia.

Patients were seen every 2 weeks; during the visits, a physical examination, blood counts and chemistries, PSA level, and adverse events were assessed. Adverse events were collected and graded according to the National Cancer Institute Common Terminology Criteria v4. Radiologic evaluation of tumor burdens was performed every 8 weeks by computed tomography (CT) scans and bone scintigraphy or using the same tests that were used for initial staging. If a patient had bone-only metastases, radiologic responses were categorized as complete response, stable disease, or progressive disease. After completion of the study treatment, these assessments and administration of ADT were performed according to the institutional guidelines.

\section{Statistics}

The primary endpoint of the study was mCRPC-free survival. The development of mCRPC was defined in accordance with the Prostate Cancer Working Group 2 (PCWG2) criteria [12]. In brief, progressive disease in the setting of medical or surgical castration was defined by one or more of the followings: (1) PSA progression defined by a minimum of 2 rising PSA values from 3 consecutive tests; (2) nodal and/or visceral disease progression as defined by RECIST; (3) bone progression defined by 2 or more new lesions. In addition, if a patient discontinued docetaxel and started a new systemic therapy for any reasons or died from any causes, whichever occurred first, the date was recorded and it was considered an $\mathrm{MCRPC}$ event. Secondary endpoints included PSA response, radiologic response, and safety. PSA response was defined as a $50 \%$ or more decline in serum PSA from baseline. Those who received at least one dose of study treatment and had follow-up were assessed for safety. A follow-up duration was calculated from the date of the study enrollment until death or the last follow-up. Time for follow-up and survival were calculated using the Kaplan-Meier method. A Cox proportional hazards regression model was used to identify independent factors associated with prolonged mCPRC-free 
survival. Variables for regression analysis included age, ECOG performance status, disease status, previous therapy, Gleason score, presence of visceral metastasis, or baseline PSA. Visual assessment of Kaplan-Meier method was used to verify the PH assumption. Twosided $P$ values less than 0.05 were considered to indicate significance. All statistical analyses were performed using SPSS v.18 for Windows.

Sample size calculation is based on single-stage phase II designs. Detected between 40 and 60\% CRPC-free rates at one-sided significance level of $0.05,1-\beta$ power
0.80 . A study requires 42 subjects to decide whether the proportion responding, $\mathrm{P}$, is less than or equal to 0.400 or greater than or equal to 0.600 . If the number of responses is 23 or more, the hypothesis that $P<=0.400$ is rejected with a target error rate of 0.050 and an actual error rate of 0.038 . If the number of responses is 22 or less, the hypothesis that $P>=0.600$ is rejected with a target error rate of 0.200 and an actual error rate of 0.197. Assuming $10 \%$ drop-out, it is planned to recruit up to 47 patients in for this study. The final efficacy

Table 1 Baseline Characteristics of the Total Population

\begin{tabular}{|c|c|}
\hline Baseline Characteristics & $N=42(\%)$ \\
\hline Age, years, median (range) & $68(55-80)$ \\
\hline \multicolumn{2}{|l|}{ ECOG performance status } \\
\hline 0 & $12(29)$ \\
\hline 1 & $30(71)$ \\
\hline \multicolumn{2}{|l|}{ Disease status } \\
\hline Recurrent after local therapy & $6(14)$ \\
\hline Initially metastatic & $36(86)$ \\
\hline \multicolumn{2}{|l|}{ Previous treatment } \\
\hline Prostatectomy & $5(12)$ \\
\hline Prostate radiotherapy & $4(10)$ \\
\hline \multicolumn{2}{|l|}{ Gleason Score } \\
\hline 7 & $3(7)$ \\
\hline 8 & $14(33)$ \\
\hline 9 & $23(55)$ \\
\hline 10 & $2(5)$ \\
\hline \multicolumn{2}{|l|}{ Metastasis } \\
\hline Bone & $34(81)$ \\
\hline Lymph node & $24(57)$ \\
\hline Visceral & $15(36)$ \\
\hline * Bone+Lymph node & $14(33)$ \\
\hline${ }^{*}$ Bone+Lymph node+Visceral & $5(12)$ \\
\hline \multicolumn{2}{|l|}{ Number of metastatic sites } \\
\hline 1 & $19(45)$ \\
\hline 2 & $16(38)$ \\
\hline 3 or more & $7(17)$ \\
\hline \multicolumn{2}{|c|}{ Volume of metastases (according to CHAARTED trial) [3] } \\
\hline Low & $8(19)$ \\
\hline High & $34(81)$ \\
\hline \multicolumn{2}{|c|}{ Risk of metastases (according to LATITUDE trial) [5] } \\
\hline Low & $12(29)$ \\
\hline High & $30(71)$ \\
\hline \multicolumn{2}{|l|}{ Baseline Laboratory Finding, Median (range) } \\
\hline Hemoglobin, g/dl & $12.75(8.5-17.5)$ \\
\hline Alkaline phosphatase, U/L & $1570(450-3070)$ \\
\hline Absolute neutrophil count, $\times 10^{3} / \mu \mathrm{L}$ & $3270(1770-7880)$ \\
\hline Neutrophil lymphocyte ratio & $2.15(0.8-6.1)$ \\
\hline PSA at screening, $\mathrm{ng} / \mathrm{ml}$ & $66.90(0.04-2759.4$ \\
\hline
\end{tabular}

ECOG Eastern Cooperative Oncology Group, PSA Prostate specific antigen

*Because patients could have metastases at multiple sites, the total numbers of metastases are larger than the number of patients 
analysis will be performed according to the intent-totreat (ITT) population.

\section{Results}

Between Aug. 2018 and Sep. 2019, a total of 42 patients entered the study and received first-line docetaxel plus ADT. Baseline characteristics of patients are summarized in Table 1 . The median age at study entry was 68 years (range 55-80). Most (86\%) patients had mCNPC at initial diagnosis, while other patients $(14 \%)$ had local disease at initial diagnosis but experienced disease progression despite local treatment. These patients were enrolled to current study at the time of mCNPC diagnosis. The median Gleason score was 9 (range 7-10). Bone metastases were noted in 34 (81\%) patients, and the median number of sites of bone lesions was 2 (range 2-6). Fifteen (36\%) patients had visceral metastases, with lung $(n=10)$ and liver $(n=5)$ being the most frequent sites.

\section{Safety}

Of the 42 enrolled patients, 36 (86\%) completed the planned 12 cycles of docetaxel plus ADT: 3 patients discontinued because of disease progression and 3 because of adverse events (pneumonitis, $n=2$; death, $n=1$ ). A 73-year-old patient died following a fall in his home shortly after the fifth cycle of docetaxel. Biweekly docetaxel was generally well tolerated (Tables 2). The most commonly observed adverse events of any grade were anemia (95\%), nail changes (33\%), fatigue (29\%), and oral mucositis $(26 \%)$. Severe (grade 3 or higher) adverse events were infrequent: pneumonitis $(n=2)$, diarrhea $(n=1)$, and neutropenia $(n=1)$. Any case of febrile neutropenia had not been reported until data cutoff date. Among the 465 cycles of biweekly docetaxel for all patients, 18 and 19 doses were delayed and reduced, respectively. As a dose intensity of docetaxel $20 \mathrm{mg} / \mathrm{m}^{2} /$ week was planned, the relative dose intensity was $98 \%$ (95\% confidence interval [CI] 86-100).

\section{Outcomes}

All but two patients (95\%) achieved a PSA response (Table 3). After 6 months of biweekly docetaxel, a median PSA nadir of $0.42 \mathrm{ng} / \mathrm{ml}$ (range $0.01-1280.87$ ) was achieved. Of note, a decrease in PSA was observed in all but one patient. (Fig. 1) A 65-year-old man with multiple bone and lymph node metastases showed a rapid deterioration of symptoms and PSA progression after 2 cycles. A retrospective review of his tumor biopsy revealed a Gleason score $9(5+4)$ adenocarcinoma in 12/12 cores with ATM (G494D) somatic mutation. Among the 31 patients who had measurable disease at baseline, 29 had an objective response per RECIST. In an
Table 2 Treatment-Related Adverse Events

\begin{tabular}{|c|c|c|c|c|}
\hline Adverse events & All Grades & $\%$ & Grade $\geq 3$ & $\%$ \\
\hline Nail change & 14 & 33.3 & 0 & \\
\hline Fatigue & 12 & 28.6 & 0 & \\
\hline Mucositis & 11 & 26.2 & 0 & \\
\hline Alopecia & 9 & 21.4 & 0 & \\
\hline Neuropathy & 8 & 19.0 & 0 & \\
\hline Constipation & 7 & 19.0 & 0 & \\
\hline Pain & 7 & 16.7 & 0 & \\
\hline Insomnia & 6 & 14.3 & 0 & \\
\hline Rash & 5 & 11.9 & 0 & \\
\hline Anorexia & 5 & 11.9 & 0 & \\
\hline Lacrimation & 5 & 11.9 & 0 & \\
\hline Dizziness & 3 & 7.1 & 0 & \\
\hline Diarrhea & 3 & 7.1 & 1 & 2.4 \\
\hline Localized Edema & 2 & 4.8 & 0 & \\
\hline Chest discomfort & 2 & 4.8 & 0 & \\
\hline Pruritus & 2 & 4.8 & 0 & \\
\hline Dyspnea & 2 & 4.8 & 0 & \\
\hline Facial flushing & 2 & 4.8 & 0 & \\
\hline Pneumonitis & 2 & 4.8 & 2 & 4.8 \\
\hline Nocturia & 1 & 2.4 & 0 & \\
\hline Myalgia & 1 & 2.4 & 0 & \\
\hline Sore throat & 1 & 2.4 & 0 & \\
\hline Anemia & 40 & 95.2 & 0 & \\
\hline Lymphopenia & 13 & 31 & 2 & 4.8 \\
\hline Neutropenia & 11 & 26.2 & 1 & 2.4 \\
\hline Thrombocytopenia & 8 & 19 & 0 & \\
\hline AST elevation & 1 & 2.4 & 0 & \\
\hline ALT elevation & 1 & 2.4 & 0 & \\
\hline
\end{tabular}

AST aspartate aminotransferase, $A L T$ alanine aminotransferase

intent-to-treat principle, the response rate was $69 \%$ (95\% CI 55-83). An additional 11 patients had stable disease, leading to a disease control rate of $95 \%$. With a median follow-up of 25 months, the median mCRPC-free survival was 26.4 months (95\% CI 20.9-32.0, Fig. 2). The one-year mCRPC-free rate was 79\% (33 patients, 95\% CI 66-91).

Table 3 Treatment outcomes of biweekly Docetaxel plus ADT

\begin{tabular}{ll}
\hline Treatment outcomes & $\boldsymbol{N}=\mathbf{4 2}(\%)$ \\
\hline PSA response at 12 weeks & \\
$\geq 30 \%$ decline & $\mathbf{4 0}(\mathbf{9 5 . 2 \% )}$ \\
$\geq 50 \%$ decline & $\mathbf{4 0}(\mathbf{9 5 . 2} \%)$ \\
$\geq 90 \%$ decline & $\mathbf{2 8}(\mathbf{6 6 . 7 \% )}$ \\
Objective Response Rate & $\mathbf{6 9 \%}$ \\
mCRPC-free survival, Median $(95 \% \mathrm{Cl})$ & $\mathbf{2 6 . 4}$ months $(\mathbf{2 0 . 9}-\mathbf{3 2 . 0})$ \\
Overall Survival, Median $(95 \% \mathrm{Cl})$ & Not reached
\end{tabular}

$M C R P C$ metastatic castration resistant prostate cancer, $\mathrm{Cl}$ confidence interval 


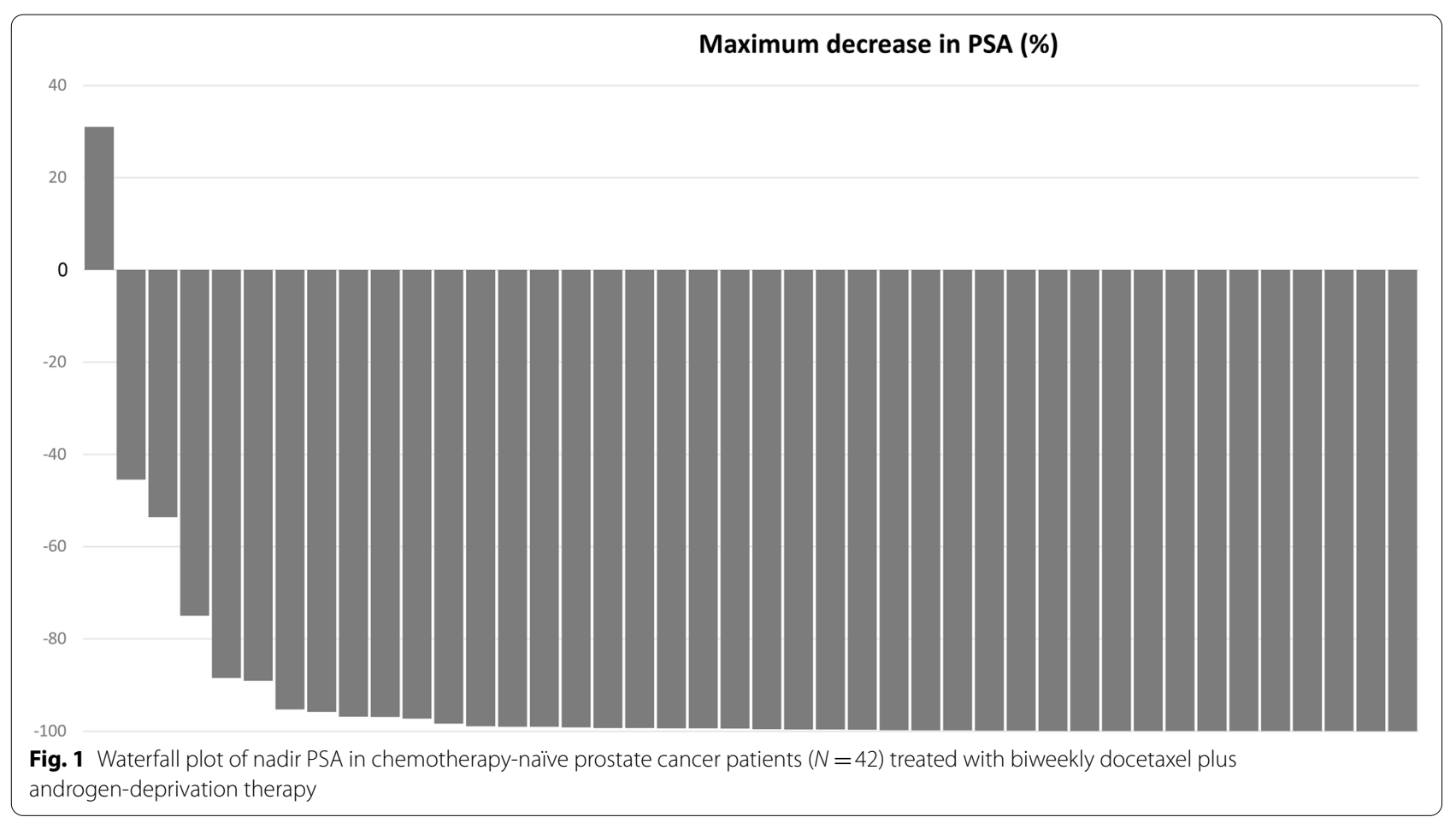

To explore predictive factors for a prolonged mCRPCfree survival with biweekly docetaxel plus ADT, we performed a multivariable regression analysis using known clinical and laboratory parameters. While mCRPC-free survival was independently associated with an ECOG performance status of 0 (hazard ratio [HR] 0.27, 95\% CI $0.07-0.99)$, it was not influenced by age, disease status, previous therapy, Gleason score, presence of visceral metastasis, or baseline PSA. Further analysis regarding mCRPC development modified by interaction between parameters did not find significant interaction.

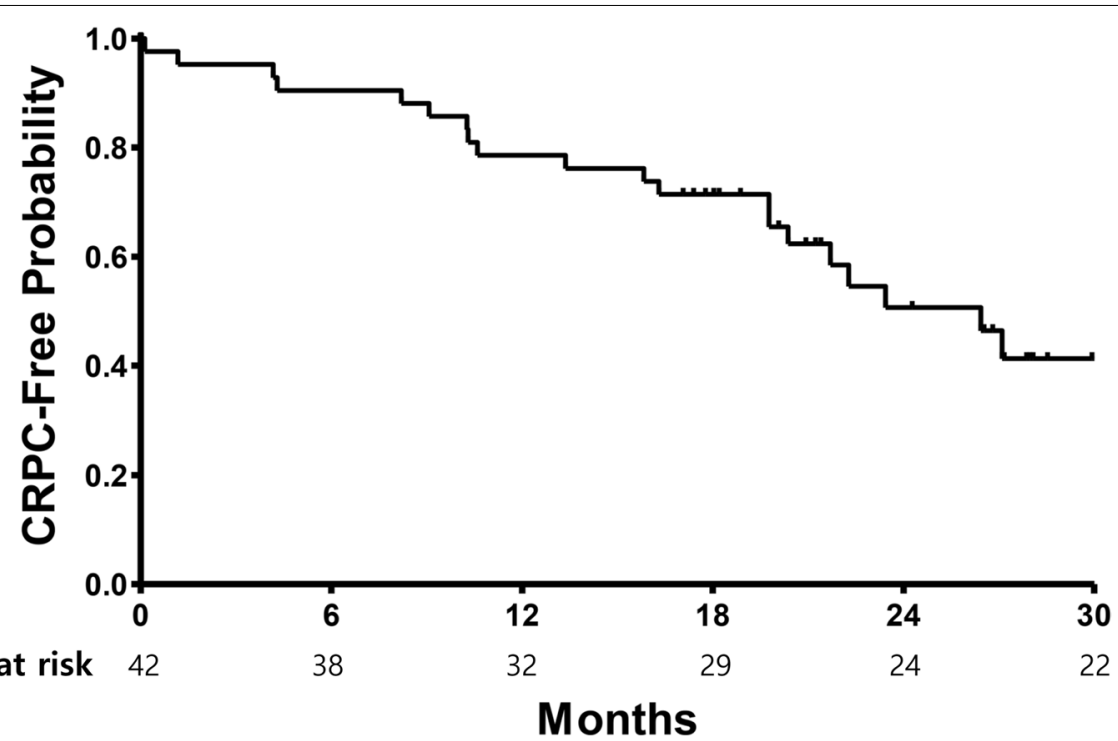

Fig. 2 Kaplan-Meier curves for CRPC-free survival of chemotherapy-naïve prostate cancer patients $(N=42)$ treated with biweekly docetaxel plus androgen-deprivation therapy. CRPC: castration resistant prostate cancer 


\section{Discussion}

The present prospective phase II study of biweekly docetaxel $(40 \mathrm{mg} / \mathrm{m} 2)$ plus ADT for the treatment of $\mathrm{mCNPC}$ shows the feasibility of using a more frequent, but lower dosing docetaxel treatment with clinical outcomes consistent with previous phase III trials conducted with the standard docetaxel regimen. PSA (95\%) and radiologic (69\%) responses were encouraging, and the toxicity profile was mild and easily manageable. The results compared well with our previous retrospective study [11], where the same docetaxel regimen was used in the treatment of high-risk $\mathrm{mCNPC}$.

In the treatment of $\mathrm{mCNPC}$, we already have more than a few treatment options including ADT plus docetaxel, abiraterone acetate, apalutamide, and enzalutamide $[6,7,13]$. Although no direct comparisons have been conducted between the available options for mCNPC, STAMPEDE investigators provided results from a retrospective analysis of 566 patients who had been treated in the docetaxel and the abiraterone arms in the overlapping period from Nov 2011 and Mar 2013 [14]. With a median follow-up of 4 years, they found no difference in prostate cancer-specific survival (HR 1.02, 95\% CI 0.70-1.49) between the two groups. Although abiraterone treatment showed better failure-free survival (HR 0.51, 95\% CI 0.39-0.67) and progression-free survival (HR 0.65, 95\% CI 0.48-0.88), OS was favored docetaxel treatment (HR 1.16, 95\% CI 0.82-1.65). This discrepancy might be originated from larger number of non-cancer related deaths in the abiraterone arm than docetaxel arm (19\% versus 9\%, respectively).

One of the main differences between docetaxel and abiraterone or androgen receptor-targeting agents was the treatment duration. Patients with $\mathrm{mCNPC}$ receive ADT plus either $<6$ months of docetaxel or long-term (i.e., until progression or unacceptable toxicities) abiraterone. If a patient with $\mathrm{mCNPC}$ receives ADT plus abiraterone or enzalutamide, treatment has to be continued for years, during which the patient is constantly exposed to therapy and thus more prone to cumulative toxicity. Conversely, if a patient receives ADT plus docetaxel, acute hematologic toxicities can sometimes be severe [3, 4]. A real-world, retrospective study showed that the incidence of febrile neutropenia was $18 \%$, and docetaxel dose reductions and delays were required in 39 and $16 \%$ of cases. The rates were even higher in Asian patients, representing $64-97 \%$ of patients treated with docetaxel plus ADT experienced grade $\geq 3$ neutropenia $[15,16]$. Considering more than half of cases are diagnosed in elderly patients, hematologic toxicities of docetaxel can be a major hurdle for general application to mCNPC. In the present study, we tested docetaxel at a lower $\left(20 \mathrm{mg} / \mathrm{m}^{2} /\right.$ week $)$ dose, based on a pharmacokinetic study conducted in Japan [17] and the belief that safety and tolerability are indispensable in the treatment of cancer patients in a palliative setting [18]. As expected, our biweekly regimen was welltolerated and appeared effective in the treatment of mCNPC.

There are several limitations in this study, including being a single-center trial with a small sample size. Additionally, our results are limited to $\mathrm{MCNPC}$ patients with high-volume disease. Although the study protocol permitted inclusion of all patients with mCNPC irrespective of risk group, recruitment through a multidisciplinary tumor board led to inclusion of patients with extensive bone and/or visceral metastases. Despite these limitations, the prospective study design and demonstration of comparable clinical efficacy support consideration of the biweekly $40 \mathrm{mg} / \mathrm{m}^{2}$ schedule for patients with $\mathrm{mCNPC}$ treated with ADT plus docetaxel.

\section{Conclusion}

Docetaxel administered $75 \mathrm{mg} / \mathrm{m}^{2}$ every 3 weeks is a current standard regimen in the treatment of mCNPC. However, treatment-related adverse events are common in elderly and fragile patients who receive the standard regimen. Biweekly $40 \mathrm{mg} / \mathrm{m}^{2}$ docetaxel plus ADT is feasible, and clinical efficacy does not seem to be compromised. Our results suggest that implementation of this approach in select patients may result in reduced toxicity, improved quality of life, and potentially improved clinical outcomes.

\section{Acknowledgements \\ The authors thank all of the patients and their families for their contributions to this study.}

\section{Authors' contributions}

SB, HK and SHP designed the study concept, and drafted the manuscript. HGJ and SIS collected the data. SSJ and HML analyzed the data. SIL reviewed the manuscript. All authors contributed to writing the manuscript and approved the final version.

\section{Funding}

This investigator-sponsored study was funded by Sanofi-Aventis Korea (Seoul, Korea). The study drug (docetaxel, Taxotere ${ }^{\mathrm{TM}}$ ) was kindly provided by SanofiAventis Korea.

\section{Availability of data and materials}

The datasets of current study are available from the corresponding authors on reasonable request.

\section{Declarations}

Ethics approval and consent to participate

The study protocol was approved by the institutional review board of Samsung Medical Center (SMC 2017-01-005-005, Seoul, Korea). All patients provided written informed consent. All study procedures were conducted in accordance to good clinical practice, and the Declaration of Helsinki. 


\section{Consent for publication}

Not applicable.

\section{Competing interests}

The authors have no conflicts of interest to declare.

\section{Author details}

'Department of Internal Medicine, Chungbuk National University Hospital, Chungbuk National University College of Medicine, Cheongju, South Korea. ${ }^{2}$ Division of Hematology-Oncology, Department of Medicine, Sungkyunkwan University School of Medicine, Samsung Medical Center, Seoul, South Korea. ${ }^{3}$ Department of Urology, Samsung Medical Center, Sungkyunkwan University School of Medicine, Seoul, South Korea. ${ }^{4}$ Internal Medicine, Dankook University College of Medicine, Cheonan, Chungcheongnam-do, Korea.

Received: 12 April 2021 Accepted: 16 November 2021

Published online: 29 November 2021

\section{References}

1. Hong S, Won YJ, Park YR, et al. Cancer statistics in Korea: incidence, mortality, survival, and prevalence in 2017. Cancer Res Treat. 2020;52:335-50.

2. Lonergan PE, Tindall DJ. Androgen receptor signaling in prostate cancer development and progression. J Carcinog. 2011;10:20.

3. Sweeney CJ, Chen $\mathrm{YH}$, Carducci M, et al. Chemohormonal therapy in metastatic hormone-sensitive prostate Cancer. N Engl J Med. 2015;373:737-46

4. James ND, Sydes MR, Clarke NW, et al. Addition of docetaxel, zoledronic acid, or both to first-line long-term hormone therapy in prostate cancer (STAMPEDE): survival results from an adaptive, multiarm, multistage, platform randomised controlled trial. Lancet. 2016;387:1163-77.

5. Fizazi K, Tran N, Fein L, et al. Abiraterone plus prednisone in metastatic, castration-sensitive prostate Cancer. N Engl J Med. 2017;377:352-60.

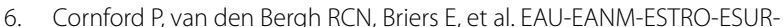
SIOG Guidelines on Prostate Cancer. Part II-2020 Update: Treatment of Relapsing and Metastatic Prostate Cancer. Eur Urol. 2021;79:263-82.

7. Parker C, Castro E, Fizazi K, et al. Prostate cancer: ESMO clinical practice guidelines for diagnosis, treatment and follow-up. Ann Oncol. 2020;31:1119-34.

8. Clarke NW, Ali A, Ingleby FC, et al. Addition of docetaxel to hormonal therapy in low- and high-burden metastatic hormone sensitive prostate cancer: long-term survival results from the STAMPEDE trial. Ann Oncol. 2019;30:1992-2003.

9. Kellokumpu-Lehtinen PL, Harmenberg U, Joensuu T, et al. 2-weekly versus 3-weekly docetaxel to treat castration-resistant advanced prostate cancer: a randomised, phase 3 trial. Lancet Oncol. 2013;14:117-24.

10. Kim HS, Lee JY, Lee SJ, et al. A retrospective feasibility study of biweekly, reduced-dose docetaxel in Asian patients with castrate-resistant, metastatic prostate cancer. BMC Urol. 2017;17:63.

11. Yoon SE, Kim Y, Cho J, et al. A retrospective feasibility study of biweekly docetaxel in patients with high-risk metastatic castration-naive prostate cancer. BMC Urol. 2019:19:30.

12. Scher HI, Halabi S, Tannock I, et al. Design and end points of clinical trials for patients with progressive prostate cancer and castrate levels of testosterone: recommendations of the prostate Cancer clinical trials working group. J Clin Oncol. 2008;26:1148-59.

13. Schaeffer E, Srinivas $S$, Antonarakis ES, et al. NCCN guidelines insights: prostate Cancer, version 1.2021. J Natl Compr Cancer Netw. 2021;19:134-43

14. Sydes MR, Spears MR, Mason MD, et al. Adding abiraterone or docetaxel to long-term hormone therapy for prostate cancer: directly randomised data from the STAMPEDE multi-arm, multi-stage platform protocol. Ann Oncol. 2018:29:1235-48

15. Tanaka N, Nishimura K, Okajima E, et al. The efficacy and safety of docetaxel-based chemotherapy combined with dexamethasone $1 \mathrm{mg}$ daily oral administration: JMTO Pca 10-01 phase II trial. Jpn J Clin Oncol. 2017:47:247-51.

16. Kwon WA, Joung JY, Lee JE, et al. Use of docetaxel plus androgen deprivation therapy for metastatic hormone-sensitive prostate cancer in Korean patients: a retrospective study. Investig Clin Urol. 2019;60:195-201.
17. Taguchi T, Furue H, Niitani H, et al. Phase I clinical trial of RP 56976 (docetaxel) a new anticancer drug. Gan To Kagaku Ryoho. 1994;21:1997-2005.

18. Lee JL, Kim JE, Ahn JH, et al. Efficacy and safety of docetaxel plus prednisolone chemotherapy for metastatic hormone-refractory prostate adenocarcinoma: single institutional study in Korea. Cancer Res Treat. 2010;42:12-7

\section{Publisher's Note}

Springer Nature remains neutral with regard to jurisdictional claims in published maps and institutional affiliations.
Ready to submit your research? Choose BMC and benefit from:

- fast, convenient online submission

- thorough peer review by experienced researchers in your field

- rapid publication on acceptance

- support for research data, including large and complex data types

- gold Open Access which fosters wider collaboration and increased citations

- maximum visibility for your research: over 100M website views per year

At BMC, research is always in progress.

Learn more biomedcentral.com/submissions 